\title{
Thomas Christensen, Stories of Tonality in the Age of François-Joseph Fétis, Chicago: University of Chicago Press 2019
}

Schlagworte/Keywords: Charles Edmond Henri de Coussemaker; François-Joseph Fétis; Gottfried Weber; Harmonielehre; music historiography; Musikgeschichtsschreibung; Raphael Kiesewetter; theory of harmony; Tonalität; tonality

Thomas Christensen muss hier nicht vorgestellt werden, handelt es sich schließlich um den Autor der Rameau-Monographie Rameau and Musical Thought in the Enlightenment ${ }^{1}$ und den Herausgeber der Cambridge History of Western Music Theory, ${ }^{2}$ um nur zwei seiner einschlägigsten Publikationen zu nennen. Zudem sind 15 seiner zahlreichen Artikel in dem Sammelband The Work of Music Theory ${ }^{3}$ erschienen. Seine Forschungstätigkeit zeugt von einem ausgeprägten und nachhaltigen Interesse am französischen Kulturraum, angefangen von seiner 1985 erschienenen Dissertation über $\mathrm{d}^{\prime}$ Alembert ${ }^{4}$ bis hin zu dem Werk, das Gegenstand dieser Rezension ist. Zu gleichen Teilen zeigt sich sein Interesse an der Geschichte des Partimento, ${ }^{5}$ welches auch das vorliegende Buch über François-Joseph Fétis insbesondere dahingehend prägt, dass die Auswirkungen dieser Praxis auch in der französischen Musiktheorie des 19. Jahrhunderts nachzuweisen versucht werden (8 f., 220, 222 f., 225 f., 261, 271).

Stories of Tonality, erschienen im Sommer 2019, ist das Ergebnis mehrjähriger Forschungsarbeit, deren Anfänge der Autor im Jahr 2008 ansetzt und für die er zwei Phasen des research leave (2011-12 und 2015-16) in Anspruch genommen hat. Es besteht aus sieben Kapiteln, die nicht chronologisch-linear, sondern nach folgenden Querschnittsthemen zusammengestellt sind:

\footnotetext{
Christensen 1993.

Christensen 2002.

Christensen 2014.

Christensen 1985.

Sanguinetti 2012, ix.
}

1. Tonal Imaginations (1-28)

2. Chant (29-66)

3. Origins (67-114)

4. Song (115-157)

5. Orienting Tonality (158-208)

6. Theory (209-244)

7. Tonal Futures (245-269).

\section{IM ZEICHEN DES STORYTELLING}

Nicht zuletzt in Anbetracht der Tatsache, dass Musiktheorie und ihre Geschichte manchen als ein abstraktes, möglicherweise sogar trockenes Thema erscheinen mag, hat sich Christensen dafür entschieden, den Stoff durch eine bewusst narrative Erzählweise zugänglich zu machen. Angesichts der Mehrdeutigkeit des französischen Begriffs histoire, der im Englischen sowohl history als auch story bedeutet (vgl. xvi), wählt er aus dem Bedeutungsspielraum eine Vorstellung von Geschichte (history) als Summe von Geschichten (stories), d. h. er rekonstruiert einen breiten Erzählstrom, der die vielen Momente von Musikgeschichte und Musiktheorie enthält, die in diesem Buch zur Sprache kommen.

Für die Darstellungsweise innerhalb des Buches bringt diese Entscheidung eine starke Aufwertung rhetorischer Mittel mit sich, was insbesondere der häufige Gebrauch von Überleitungen dokumentiert, die beim Lesen die Illusion eines Erzählflusses entstehen lassen: "But we are getting ahead of our story« (35, 39), »Most significant for our story» (78), "But let us return to our story" (96) und Ähnliches. In diesem Zuge werden die Autoren der kommentierten Texte zu Charakteren oder zu Schauspielern, wobei Fétis die Rolle des Hauptdarstellers oder vielmehr des »impresario principal« (xi) zukommt. Mehrfache Referen- 
zen auf dieselben Autoren an auseinanderliegenden Stellen im Buch werden zu Wiederbegegnungen mit bekannten Charakteren (»we met Lambillotte in chapter 2 [...]«, 69). Einige spielen nur Nebenrollen, aber andere, wie Raphael Kiesewetter oder Charles Edmond Henri de Coussemaker ${ }^{6}$ konkurrieren im Charisma mit dem Hauptdarsteller, mit dem sie unweigerlich aneinandergeraten werden.

Dank dieser literarischen Kunstgriffe liest sich Christensens Text flüssiger und fesselnder als die meisten Studien dieser Art. Vor dem Hintergrund, dass historisches Wissen aus isolierten Daten gewonnen wird, welche Historiker*innen aus gesammelten Quellen extrahieren, und dass die wissenschaftliche Verantwortung in der Auswahl der Daten, in ihrem gegenseitigen In-Beziehung-Setzen und ihrer Interpretation liegt, besteht kein Zweifel daran, dass Christensen bei dieser kritischen Arbeit ausgesprochen gründlich war, wenn auch sein Erzählstil dazu neigt, dies zugunsten eines >Realitätseffekts` (effet de réel) ${ }^{7}$ vergessen zu machen.

\section{... UND WIEDER FÉTIS}

Während seiner gesamten Laufbahn hat Fétis daran gearbeitet, sich selbst große Sichtbarkeit zu verschaffen. Seinem offensichtlichen Wissensdurst kommt nur sein ständiges Streben nach öffentlicher Anerkennung gleich. Seine unaufhörliche Publikationstätigkeit ermöglicht ihm das zu besetzen, was man heute einen smedialen Raum` nennen würde. Fétis verstand alle Vorteile zu nutzen, die er aus der Presse ziehen konnte, um sich in den Mittelpunkt der Pariser Musikwelt zu stellen: Die Revue musicale (1827-35), gefolgt von der Revue et gazette musicale ab 1835 waren für die Verbreitung seiner Ideen wesentliche Instrumente.

In einer Weise, die Fétis vermutlich gefreut hätte, hat die Musikgeschichtsschreibung des 20. und 21. Jahrhunderts diese Omnipräsenz

6 »Charles Edmond Henri de Coussemaker (1805-76) is one of the most fascinating protagonists in our story [...].«(79)

7 Ich verwende diesen Ausdruck im Sinne von Barthes 1968. Man kann ebenso an die Theorie des effet de vie denken, wie sie von Münch 2004 entwickelt wurde. des Musikschriftstellers und Theoretikers noch fortgeschrieben, indem sie ihn ins Zentrum historischer Forschung über die französischsprachige Musiktheorie des 19. Jahrhunderts rückte. ${ }^{8}$ Wie der Titel des Buches andeutet, scheint sich Christensen in dieselbe historische Perspektive einzuschreiben.

\section{KONFRONTATION ALS LEBENSFORM}

Eine solche Vorgehensweise wird dadurch erleichtert und eventuell auch nahegelegt, dass Fétis über seine gesamte Schaffenszeit hinweg in zahlreiche wissenschaftliche Diskurse involviert war, die zumeist in Form erbitterter Polemiken geführt wurden. Christensen führt mehrere Beispiele für Fétis' wissenschaftliche Kontroversen an wie z. B. die mit Kiesewetter, Coussemaker, François-Auguste Gevaert, Alexandre Joseph Vincent, Victor Derode und Auguste Barbereau. Fétis behauptet unablässig seine Ideen und verteidigt sie gegen Attacken, aber er kennt auch den >Präventivschlag`: Tatsächlich greift er systematisch jede abweichende Meinung an, die seine eigenen Theorien schwächen könnte. Der Angriff auf Derode ist ein gutes Beispiel für diese Strategie.

Es mag erstaunen, dass Fétis sich überhaupt die Mühe gemacht hat, eine Rezension über die Harmonielehre ${ }^{9}$ dieses $>$ Amateurtheoretikers` zu verfassen: Die Abhandlung ist konfus Christensen hat durchaus Recht, Derode als "pitiable writer (10) zu bezeichnen - und ein Theoretiker vom Rang Fétis' hätte sie einfach ignorieren können. Derodes Abhandlung hat jedoch immerhin ein Verdienst: Sie stellt das Dogma der Skala als Modell für die Theorie der Tonalität in Frage.

La gamme est-elle un fait primitif en musique? est-elle un principe? On appelle ainsi toute notion qui, servant à expliquer les autres, ne peut point être expliquée à son tour. Ces deux caractères manquent à la

8 Auf die überreiche Literatur zu den verschiedenen Aspekten von Fétis' Arbeiten kann hier nur kurz verwiesen werden. Eine ziemlich vollständige Bibliographie findet sich bei Campos 2013, 783-786, die nun sehr hilfreich von derjenigen aus Christensens Stories of Tonality vervollständigt wird.

9 Derode 1828. Die Rezension ist in der Revue musicale 3 (1828), 217-223 erschienen. 
gamme: elle ne peut donner la raison de rien, tandis qu'on peut au contraire rendre compte de la succession des notes qui la composent. [...] La gamme n'a rien de nécessaire dans les proportions qui la composent, rien qui soit l'essence de I'harmonie; elle ne contient pas en ellemême les principes de sa formation, rien qui puisse nous faire procéder à la former, si, par hasard, on venait à perdre le souvenir de sa marche. ${ }^{10}$

Derode stellt diese Frage zu Recht, ${ }^{11}$ auch wenn der Rest seines Traktats zeigt, dass er nicht imstande ist, die theoretischen Konsequenzen aus diesem grundsätzlichen In-FrageStellen zu entwickeln. Aber es ist genau diese Idee Derodes, die Fétis in Alarmbereitschaft versetzt. 1828 weiß er bereits, dass die Tonleiter ein zentrales Element in seinen eigenen Überlegungen zur Tonalität sein wird, die er in den Folgejahren zu entwickeln im Begriff ist. Er kann daher nicht riskieren, dass ein Autor die Grundlagen seiner eigenen Theorie in Frage stellt.

Die Kontroverse mit Barbereau ist ein weiteres Beispiel für Fétis' Strategie. Sie wurde zur öffentlichen Auseinandersetzung anlässlich der letzten einer Reihe von vier Konferenzen, die Fétis organisiert hatte, um Werbung für seinen Traité $d^{\prime}$ harmonie ${ }^{12} \mathrm{zu}$ machen $(241 \mathrm{f}$.). Fétis nutzt seine Begabung als Redner, um einen

10 Ebd., 321, 325 (»Ist die Tonleiter wirklich ein Grundelement der Musik? Ist sie ein Prinzip? So nennt man nämlich jeden Begriff, der zur Erklärung anderer dient und seinerseits wiederum nicht erklärt werden kann. Diese beiden Züge fehlen jedoch der Tonleiter: Sie kann gar nichts erklären, wohingegen man sie auf die Noten zurückführen kann, aus denen sie sich zusammensetzt. [...] Die Tonleiter hat nichts Zwingendes in den Verhältnissen, aus denen sie aufgebaut ist, nichts, was das Wesen der Harmonie ausmachen würde; sie enthält in sich selbst nicht die Prinzipien ihrer Bildung, nichts, was uns in die Lage setzen würde, sie zu bilden, falls uns zufällig die Erinnerung an ihren Verlauf abhanden käme. «)

11 Das Dogma von der Tonleiter als theoretisches Werkzeug zur Erklärung der Tonalität oder der Harmonik wird ebenso von Barbereau, Vivier und Loquin verworfen. Vgl. Rigaudière 2019, 67-69.

12 Fétis 1844. bekannten und geschätzten Theoretiker in eine schwache Position zu bringen, als dieser im Begriff ist, auch einen Traité d'harmonie zu veröffentlichen. Diese Episode, die in der Revue et gazette musicale von Maurice Schlesinger, ${ }^{13}$ dem Besitzer des Journals und dem Herausgeber der Werke Fétis', als Triumph des Letzteren verbreitet wurde, ist bezeichnend für die Macht der Presse im 19. Jahrhundert und ebenso für die Methoden, derer Fétis sich bedient, um potenzielle Konkurrenz aus dem Weg zu räumen.

In der historischen Rückschau wird deutlich, dass die Abhandlung von Barbereau, ${ }^{14}$ auch wenn sie einen ganz anderen Ansatz als Fétis verfolgt, ein reiches Werk und eben doch eine ernsthafte Konkurrenz ist. Insbesondere das Kapitel über harmonische Progressionen lässt ein bemerkenswertes präfunktionales Denken erkennen. ${ }^{15}$

Eine weitere Auseinandersetzung findet, wiederum später, mit Vincent statt (181 f.). Vincent war, wie auch andere Historiker seiner Zeit, von der Vorstellung überzeugt, dass die griechische Antike Formen der Harmonik oder der Mehrstimmigkeit gekannt haben könnte. Vincents gedruckter Vortrag ${ }^{16}$ gab einige Jahre darauf Anlass zu einer heftigen Replik durch Fétis. ${ }^{17}$ Christensen benennt die Ursachen dieser Virulenzen klar: In seinem Résumé von 1835 hatte Fétis die These verteidigt, dass die gesamte Musik des Mittelmeerraums solange monodisch geblieben war, bis die Harmonie in primitiver Form von den "peuples septentrionaux ${ }^{18}$ im 5. Jahrhundert eingeführt wurde (97-99, 179 f.). Vincents Hypothese stellte also nicht nur diese Idee grundlegend in Frage, sondern auch Fétis' Vorstellung des historischen Ursprungs von Harmonik und 'Tonalitäts an sich.

13 Revue et gazette musicale 11/9 (3.3.1844), 78.

14 Barbereau [1845].

15 Ebd., 2-39. Vgl. Rigaudière 2019, 136-138.

16 Vincent 1854.

17 Fétis 1859.

18 Zum Begriff sseptentrionalı: `Nördliche Völkerı, verweist mit dem septentrio, dem Großen Wagen, auf die nördliche Hemisphäre. (Anmerkung der Übersetzerin) 


\section{WISSENSCHAFT VOR KUNST}

Je mehr Fétis seine Ideen zu bestätigen sucht, desto mehr verteidigt er sie gegen tatsächliche oder vermeintliche Angriffe, und desto mehr zieht er sich auf seine eigenen gesicherten Positionen zurück. Christensen zeigt, dass Fétis als Theoretiker im Traité keine Skrupel hat, Passagen in den Werken großer Komponisten zu kritisieren, da sie seiner Ansicht nach gegen die Gesetze der Tonalität verstoßen (226230). ${ }^{19}$

Insbesondere kommentiert er einen 'Fehler, den Fétis im zweiten Satz von Beethovens Fünfter Sinfonie (T. 132-138) nachweist (227). ${ }^{20}$ Dieser angebliche Fehler besteht darin, die Septime eines "accord de septième sensible" (eines halbverminderten Septakkords $d$-f-as-c) - von Fétis interpretiert als "accord de septième de dominante avec substitution sdu sixième degré à la dominante« (ein durch Substitution der Dominante $b$ konstruierter Dominantseptnonakkord) - nicht im regelhaften Abstand von einer Septime zum Grundton, sondern stattdessen im Abstand von einer Sekunde unterhalb des Grundtons gesetzt zu haben (T. 133, 135, 136).

Ein interessanter Hinweis wäre hier, dass die theoretische Quelle für diese BeethovenKritik höchstwahrscheinlich in Gottfried Webers Versuch einer geordneten Theorie der Tonsetzkunst zu finden ist, ${ }^{21}$ ein Traktat, den Fétis gut kannte, und in dem diese Passage bereits als besonders vermerkt worden war. Die unterschiedliche Behandlung dieser Passage

19 Dieses Problem wird detailliert bei Campos (2013, 271-321) behandelt.

20 Fétis 1844, 9, 49. Bei Campos findet sich die detaillierte Darstellung der Ereignisse, wonach Fétis die verblüffte Reaktion des Publikums während einer Pariser Aufführung des Werks beim Hören dieser Passage anführt, um zu zeigen, dass dieser >Fehler Beethovens die Regeln der Tonalität verletzt, und zwar so weit, dass auch die musikalisch nicht gebildeten Hörer intuitiv den anomalen Charakter dieser Passage spüren; vgl. Campos 2013, 299-302.

21 Weber 1817, 167 f. Die hohe graphische Ähnlichkeit der Notenbeispiele bei Weber und Fétis - und besonders die Verwendung des Asteriskus zur Markierung der betreffenden Akkorde - lässt keinen Zweifel an dieser Annahme. bei beiden Theoretikern lässt jedoch aufschlussreiche Rückschlüsse auf ihre ebenso unterschiedliche Geisteshaltung zu. Wo Fétis sich wie ein pontifikaler Richter verhält, vertraut Weber dem Komponisten:

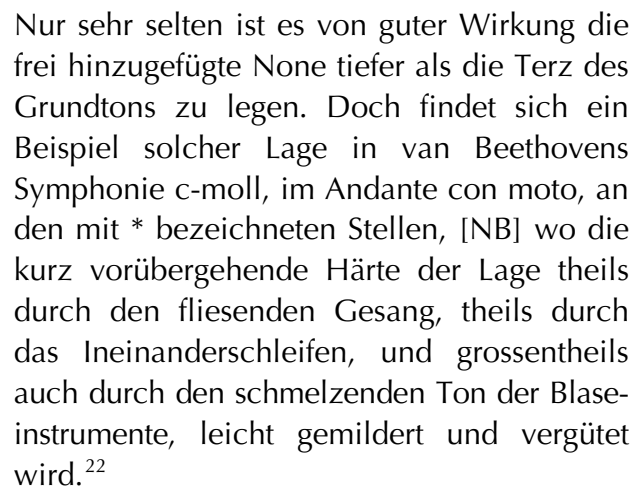

Nicht nur liegt es Weber fern, von einem Fehler zu sprechen, sondern er benennt auch Faktoren, die entstehende Dissonanzen mildern können. Er geht demnach anders als Fétis davon aus, dass Beethoven sein Handwerk vollkommen beherrscht. Es geht ihm nicht um eine Verurteilung der Passage, sondern darum, die Bedingungen zu verstehen, unter denen der Komponist eine atypische Dissonanz in Kauf nimmt.

\section{GESCHICHTE ALS ZIELORIENTIERTER PROZESS}

Rémy Campos ${ }^{23}$ hat die Anhäufung von Informationen, wie sie für Fétis charakteristisch ist, sehr präzise beschrieben. Man kann nicht umhin, Fétis' außergewöhnliche Gelehrsamkeit zu bewundern, die in der Ansammlung aller möglichen Dokumente und Objekte eine schon zwanghafte Leidenschaft verrät: Bücher, gedruckte Partituren, Zeitschriften, Manuskripte und Musikinstrumente. Dieser Appetit auf Besitz wird bekanntlich zu Vorwürfen gegen Fétis führen, Bestände der Bibliothek des Conservatoire unterschlagen zu haben. ${ }^{24}$

Fétis' Ausbildung beschränkt sich nicht nur auf Musik und Musikwissenschaft. Als Musikschriftsteller strebt er danach, auch in anderen Wissensdomänen zu Hause zu sein, und Chris-

22 Ebd., 168.

23 Campos 2013.

24 Vgl. besonders Lesure 1974-76; Fétis 2006, 12, 90, 92, 100, 102-105; Campos 2013, 52, 233. 
tensen verweist z. B. auf seinen Vortrag vor der Pariser Société d'anthropologie im Februar 1867 (193 f.). Fétis möchte mehr als nur eine große Menge an Informationen im Sinne enzyklopädischer Geschichtsschreibung zusammentragen, er will eine Geschichte im Dienste rationalen Denkens schreiben. Christensen zeigt, dass Fétis' Vision von Geschichte von seiner Hegel-Lektüre beeinflusst ist. Die Idee einer Weltgeschichte, die das Wirken des Weltgeistes im Laufe der Jahrhunderte nachzeichnet, hat Fétis vermutlich fasziniert. Nach Christensen scheint Fétis tonalité als musikalisches Analogon zum Hegel'schen Weltgeist verstanden zu haben (20).

Fétis' Umgang mit der Vergangenheit liegt offenbar eine solche teleologische Geschichtsphilosophie zugrunde. Dies gilt für sein Verständnis von Musikgeschichte, aber auch für sein Verständnis von Musiktheorie. Sein Verhältnis zu den Autoren der Vergangenheit ist dabei nicht weniger skriegerisch seinen Zeitgenossen: Er betrachtet sie mit generellem Misstrauen. Seine kritische Auseinandersetzung mit älteren Theoretikern in der Esquisse, ${ }^{25}$ dem Traité ${ }^{26}$ und in den beiden Ausgaben der Biographie universelle ${ }^{27}$ ist oft dadurch eingeschränkt, dass er ihnen vorwirft, sie hätten in die Prinzipien, die er selbst entdeckt zu haben glaubt, keinen Einblick gehabt.

Im Gegenteil, aber das ist selten, kann diese Logik auch zu einer gewissen Nachsicht führen: Georg Andreas Sorge z. B. ist einer der wenigen Theoretiker, der in seinen Augen allein dadurch Gnade findet, dass er die Möglichkeit des unvorbereiteten Eintretens bestimmter Septakkorde erkannt hat (219 f.).

Schließlich basiert seine Geschichte der Musiktheorien weitgehend auf Instrumentalisierung bis zur Karikatur reduzierter älterer Theorien in der Absicht, sich selbst als Endpunkt einer ızweiten` Teleologie zu setzen, parallel zu derjenigen, nach der sich die Entwicklung der Tonalität in der Geschichte vollzog.

25 Fétis 1840.

26 Fétis 1844.

27 Fétis 1835-44 und 1860-67.

\section{DIE GESCHICHTEN... UND DIE ,TONALITÄTEN}

Ebenso wie Christensen die Pluralität der Geschichten von Tonalität durch die Grammatik hervorhebt, hätte er auch im zweiten Teil des Buchtitels den Plural verwenden können, denn die Geschichten, die er im Buch versammelt, erzählen tatsächlich von `Tonalitäten`. Dies ist zweifellos eines der bemerkenswertesten Charakteristika dieses Buches, das es radikal von anderen Arbeiten unterscheidet, die sich bisher dem Phänomen Tonalität gewidmet haben.

Christensen erweitert das Forschungsfeld ganz erheblich, indem er keine der Bedeutungen ausschließt, die der französische Begriff tonalité im 19. Jahrhundert haben konnte, insbesondere in seinen zahlreichen Erweiterungen durch Adjektive oder andere Attribute: tonalité du plain-chant, tonalité antique, ancienne, grégorienne, ecclésiastique, moderne, vulgaire. Fétis, als ıHeld der Geschichte`, unternahm nichts, um die Mehrdeutigkeit des Begriffs zu reduzieren. Im Gegenteil, er beförderte selbst die Bedeutungsexplosion, indem er nicht nur die Opposition von tonalité ancienne und tonalité moderne aufgriff und zu seinem Thema machte, sondern auch mit seiner Idee der vier „Ordnungen« von Tonalität (unitonique, transitonique, pluritonique und omnitonique ${ }^{28}$ ein zusätzliches Kategoriensystem einführte. In gewisser Weise hat er damit zur konzeptionellen Verwirrung zwischen tonalen Ordnungssystemen beigetragen, die doch eher wenig miteinander gemein haben.

Der Umfang des Forschungsgebiets, den Christensens semantische Wahl impliziert, ist immens, und die reichhaltige Bibliographie zeigt, dass der Autor eine wahre wissenschaftliche Odyssee auf sich genommen hat. Drei von den sieben Kapiteln des Buches sind Forschungsgebieten gewidmet, die in der Historiographie des 19. Jahrhunderts traditionell nicht miteinander verbunden sind: Die Reform des gregorianischen Gesangs, das traditionelle Repertoire der verschiedenen französischen Regionen und außereuropäisches Repertoire.

Die tonalité du plain-chant (Tonalität des einstimmigen, in der Regel gregorianischen Gesangs) wird im zweiten Kapitel behandelt (29-66).

Fétis 1844, »Livre troisième«. 
Von plaint-chant in dieser Epoche zu sprechen, heißt, die >Akte seiner Wiederentdeckung und die Debatten um die Möglichkeiten seiner Begleitung durch die Orgel erneut zu öffnen. Christensen dokumentiert im Detail die unterschiedlichen Positionen zwischen dem Wunsch nach Reinheit des plain-chant (Alexandre-Étienne Choron, Félix Danjou, Joseph d'Ortigue), der von der tonalité moderne infiziert gewesen sei, und der gegenteiligen Überzeugung, dass in einigen Fällen chromatische Leitton-Wirkungen einzuführen seien. Überraschenderweise vertritt Fétis den zweiten Standpunkt, indem er sich auf mehrere Passagen aus Guido von Arezzos Micrologus beruft, ${ }^{29}$ insbesondere auf einen Abschnitt über die subductio (44, 288, Anm. 4244), den er jedoch als Interpolation aus der für Martin Gerberts Ausgabe verwendeten, eher späten Abschrift entlarvt (43-48).

Auch hinsichtlich des accompagnement analysiert Christensen die verschiedenen theoretischen Positionen gründlich: Es zeigt sich, dass das Eintreten von Niedermeyer und d'Ortigue ${ }^{30}$ für eine rein diatonische Begleitung, die sich ohne Akzidentien auf die Noten des Modus beschränkt, bei weitem nicht die vorherrschende war. Tatsächlich herrschte die gegenteilige Auffassung vor, wonach der plaint-chant in der tonalité moderne harmonisiert werden kann. Dies wird von Theoretikern und Komponisten wie Jacques-Louis Battmann, ${ }^{31}$ Adrien de la Fage, ${ }^{32}$ François-Auguste Gevaert, ${ }^{33}$ Hector Berlioz ${ }^{34}$ und Théodore Nisard ${ }^{35}$ vertreten (63-66).

Das Volkslied wird in Kapitel 4 behandelt. Bevor Christensen sich der Folklore zuwendet, widmet er zunächst einen Abschnitt der Sammlung Cris de Paris (1857) von Georges Kastner (115-124). ${ }^{36}$ Die große Mühe, die Kastner der

29 Gerbert 1784.

30 Niedermeyer/d'Ortigue 1855.

31 Battmann 1855.

32 La Fage 1855-56.

33 Gevaert 1856.

34 Berlioz 1862.

35 Nisard 1860

36 Kastner 1857. Die cris de Paris waren eine verbreitete Quelle der Inspiration vom 16. bis 19. Jahrhundert, sei es in der Literatur, der bildenden Kunst oder in der Musik. Vgl. Milliot 2016. akribischen Transkription der cris (Rufe, Schreie) gewidmet hat, also der Rufe, die die verschiedenen Metiers kennzeichnen, kann man nur bewundern, allerdings veranlasst es heute fast zum Schmunzeln, dass er alle diese tönenden Signale, die einem stark modulierenden parlando näher gewesen sein müssen als veritablem Gesang, mit präziser Melodik und Intonation zu notieren versucht hat. Vor der Erfindung der Tonaufnahme war Kastners Notation jedoch die einzige Möglichkeit, diese klanglichen Manifestationen des Pariser petit peuple wenigstens annähernd zu fixieren. Man kommt nicht umhin, an die Akribie zu denken, mit der Olivier Messiaen ein Jahrhundert später Vogelgesänge aufzeichnen wird.

In Bezug auf das Volkslied präzisiert Christensen, dass das Sammeln von Liedgut in Frankreich erst in den 1830er Jahren Gegenstand »konzertierter Bemühungen« (»concerted efforts", 125) war. Ebenso erinnert er an die politischen Beweggründe für die von Napoleon III. eingeleiteten Maßnahmen zur Sammlung französischer Volksmusik. Diese Förderung des Folklorismus war eine wichtige Unterstützung für die Förderung konservativer und nationalistischer Werte. (Ebd.) Die Untersuchung der tonalité dieses Repertoires stützt sich auf zwei Ausgangshypothesen: Entweder beruht sie auf gregorianischer Modalität (Abbé Jean Guillaume Herry, ${ }^{37}$ Désiré Beaulieu ${ }^{38}$ ) oder auf einem $` Z u-$ sammenleben ‘ von tonalité ancienne und tonalité moderne (Coussemaker, ${ }^{39}$ Jean-Baptiste Weckerlin, ${ }^{40}$ Julien Tiersot ${ }^{41}$ ).

Louis-Albert Bourgault-Ducoudray ${ }^{42}$ sucht als einziger in der griechischen Antike nach einer Quelle für den Volksgesang (135-138). Das Fehlen der note sensible [der leittönig erhöhten Paenultima] wird von vielen Volksliedforschern als Zeichen von Authentizität erachtet (134).

Im fünften Kapitel wird schließlich die auBereuropäische tonalité diskutiert (158-208). Ausgehend vom Ägyptenfeldzug Napoleons I.

37 Herry 1842.

38 Beaulieu 1858.

39 Coussemaker 1855.

40 Weckerlin 1886

41 Tiersot 1889.

42 Bourgault-Ducoudray [1876]. 
befasst sich Christensen mit der Geschichte der Orientalismus-Studien in der Musikwissenschaft und hebt die wichtigsten Fragen hervor, auf die sich diese Studien konzentrieren. Die erste Frage ist die nach den Ursprüngen der mündlich überlieferten und manchmal auch notierten Musik. Zu diesem Thema gibt es zwei konkurrierende Hypothesen. Guillaume-André Villoteau, der Musiker, den Napoleon dem wissenschaftlichen Team der Expedition beitreten ließ, sieht eine Verbindung zwischen der Theorie der arabischen Autoren, die er gelesen hatte, und den Theorien der griechischen Antike (164). Fétis, der sich auf die musikalische Ikonographie stützt, glaubt, dass der Ursprung der arabischen Musik im alten Ägypten zu suchen sei. Durch das Studium der bei Johannes Damascenus überlieferten Notenschrift, die Zeichen einer demotischen Schrift enthält, nimmt er an, dass diese aus ägyptischer Tradition stammen, und schließt daraus, dass die Musik der byzantinischen Kirche ein ziemlich getreues Abbild der Musik des alten Ägypten sein müsse (170). ${ }^{43}$ Drei Jahre später wurde diese Hypothese von Kiesewetter kategorisch dementiert, wobei er auch Fétis' Lesart der musikalischen Ikonographie in Frage stellte (174). ${ }^{44}$

Eine weitere Frage ist die nach den Mikrointervallen. Sie bringt Fétis, der von der strukturellen Präsenz von Dritteltönen in der arabischen Musik überzeugt ist, erneut in Opposition zu Kiesewetter, der die arabische Musik im Wesentlichen für diatonisch hält.

Der letzte Abschnitt dieses Kapitels (»The racialization of tonality«) bestätigt, was bereits in anderen Passagen des Buchs aufscheint: Der Entwurf der Musikgeschichte, wie Fétis ihn in seiner Histoire générale zeichnet, ${ }^{45}$ impliziert durch die Einbeziehung einer ethnologischen Dimension gleichzeitig eine offen rassistische Ideologie, inspiriert von Joseph Arthur de Gobineaus Essai sur l'inégalité des races, ${ }^{46}$ von dem Fétis ein stark kommentiertes Exemplar besaß (204). Für Fétis sind die verschiedenen races` hinsichtlich der Musik sehr ungleich

43 Fétis 1835, Ixx-Ixxiii.

44 Kiesewetter 1838.

45 Fétis 1869-76.

46 Gobineau 1853-55. und die »race blanche« (ebd.) sei die einzige, der es gelungen sei, ein ausgeklügeltes Tonsystem zu entwickeln, das einen reichen künstlerischen Ausdruck ermöglichte.

\section{DIE TONALITÉ MODERNE}

Die alten und außereuropäischen `Tonalitäten sind in dem Werk gut vertreten, aber auch die tonalité moderne wird nach Fétis' Konzeption (in Kapitel 1 und 6) diskutiert. Hier sollen allerdings nur deren Hauptmerkmale erwähnt werden, weil sie in der musikwissenschaftlichen Literatur bereits ausführlich kommentiert wurden. Wie Fétis während seiner gesamten Laufbahn nicht müde wurde zu wiederholen, ${ }^{47}$ lehnt seine Theorie jeden physikalischen oder mathematischen Determinismus ab zugunsten »metaphysischer «, ${ }^{48}$ d. h. von den musikalischen Wahrnehmungsfähigkeiten des Menschen abhängiger Gesetze, die wiederum von der sraces abhängen.

Dies erklärt, warum es verschiedene Typen tonaler Organisation je nach Epoche und Region gegeben hat und noch gibt. Für Fétis ist das motorische Potential (le potentiel de motricité), das im Intervall der verminderten Quinte oder der übermäßigen Quarte liegt, wenn 4. und 7. Tonleiterstufe kombiniert werden, ein wesentlicher Faktor der tonalité moderne (12).

In der Tat ruft diese "appellative Konsonanz ${ }^{49}$ in den Zuhörenden die Erwartung einer Auflösung hervor. Für Fétis war es daher von Interesse, den Wendepunkt in der Geschichte zu finden, an dem die Komponisten begannen, dieses Intervall bewusst einzusetzen, und zwar nicht mehr im Kontext kontrapunktischer Mechanik von Dissonanzvorbereitung und -auflösung, sondern durchaus im Rahmen eines Akkords ohne Vorbereitung, nämlich des Dominantseptakkords. Indem Fétis Monteverdi diese privilegierte Rolle zuwies (21 f., 67-69), ${ }^{50}$ gab er Anlass zu zahlreichen Debatten, was von Christensen genau verfolgt und kommentiert wird.

47 Vgl. z. B. Fétis 1840, 169 f. oder Fétis 1844, ij.

48 Dieser Begriff taucht wieder bei Momigny auf. Vgl. Groth 1983, 58.

49 Fétis 1844, 9.

50 Vgl. zum Beispiel Fétis 1840, Bd. 6, 449. 
Monteverdis 'Entdeckung`, die die Ankunft der tonalité moderne markiert, führt nach Fétis auch den Übergang zum ordre transitonique herbei. ${ }^{51}$ Der Dominantseptakkord, der zur Bestimmung einer Tonart hinreichend ist, ermöglicht tatsächlich einen leichteren Übergang von einer Tonart zur anderen (249).

\section{>TONALITÄTEN U UND SKALA}

Das weite Begriffsfeld von `Tonalität im Frankreich des 19. Jahrhunderts erklärt sich durch eine theoretische Annäherung an die Systematik der Tonordnung, welche grundsätzlich mit dem Begriff der Skala verbunden ist. Die Musikwissenschaft des 20. Jahrhunderts hat, flankiert von der Ethnomusikologie, diese verschiedenen Organisationstypen nach ihrer jeweiligen Funktionalität kategorisiert: Man kann demnach einstimmige modale Strukturen, modale Polyphonie und harmonische Tonalität nicht mit denselben theoretischen Werkzeugen erklären.

Zur Zeit von Fétis beruhten tonale Konzepte weitgehend auf verschiedenen Typen skalarer Modelle. ${ }^{52}$ Auffallend ist, dass im gesamten Buch sämtliche theoretischen und historischen Diskussionen über verschiedene Typen von Tonalität ohne Ausnahme auf den Begriff der Skala zurückkommen, sei es, um das französische Volkslied zu beschreiben, außereuropäische Musik, den gregorianischen Gesang, die mittelalterliche Mehrstimmigkeit oder die der Renaissance. Darüber hinaus scheint sich ein Großteil dieser Debatten über bestimmtes Repertoire (plaint-chant und Volkslied) auf das Vorhandensein oder Fehlen des Leittons zu konzentrieren, als wäre dies das wichtigste Unterscheidungskriterium zwischen den verschiedenen `Tonalitäten`.

\section{ENTWICKLUNG DER TONALITÄT IN DER ZWEITEN HÄLFTE DES 19. JAHRHUNDERTS}

Das Buch endet mit der Entwicklung der Tonalität in der zweiten Hälfte des 19. Jahrhunderts und mit Fétis' Befürchtungen angesichts der weiteren Entwicklung (Kapitel 7, 245-269). Das letzte Evolutionsstadium der Tonalität ist

51 Fétis 1844, 179.

52 Vgl. Rigaudière 2019, 64-67. der ordre omnitonique, in dem, wie Christensen schreibt (252), »jede Note eines Akkords oder seine Alteration das Potential hat, Leitton zu einer neuen Tonart oder ihrer Dominante zu werden. ${ }^{53}$

Fétis betrachtet die Zukunft der Tonalität mit Pessimismus. Er befürchtet, dass die Generalisierung der enharmonie transcendante zu einem Niedergang der Musik führen wird (255). In einem der Abschnitte dieses Kapitels (259262) zeigt sich sein Desinteresse an Wagners Harmonik, die ihm doch viele Beispiele zur Ausstattung des ordre omnitonique hätte bieten können. Tatsächlich scheint Fétis nur die frühen Werke Wagners gekannt zu haben (259). Es fragt sich, ob er es womöglich vermieden hat, sich mit dieser Musik auseinanderzusetzen, weil er Schwierigkeiten hatte, ihre Harmonik auf der Grundlage seiner Theorie zu analysieren.

Betrachtet man den Abschnitt des Traité, der der enharmonie transcendante gewidmet ist, ${ }^{54}$ stellt man nicht nur fest, dass Fétis' selbstgeschriebene Beispiele unbeholfen sind, ${ }^{55}$ sondern auch, dass er seinen fiktiven Beispielen keine aus der musikalischen Literatur seiner Zeit gegenüberstellt. In den zahlreichen Neuauflagen des Traité wird Fétis niemals eine Aktualisierung dieser Passage in Angriff nehmen. Zu seiner Verteidigung lässt sich präzisierend anführen, dass Chromatik und Enharmonik in der französischen Musiktheorie erst nach und nach behandelt wurden. François-Auguste Gevaert war 1907 der erste Theoretiker des frankophonen Raums, der eine vertiefte Studie auf der Grundlage realer Literaturbeispiele vorlegte. ${ }^{56} \mathrm{Im}$ Gegensatz zu Gevaert, der eine Erweiterung des Tonmaterials auf 17 Töne in Form des chroma intégral vorschlug, ${ }^{57}$ hielt Fétis während seiner gesamten Laufbahn an der diatonischen Skala als Basis fest, obwohl sie sich als unzureichend für eine Theorie chromatischer Harmonik erwiesen hatte.

53 »In practice, this seems to mean that any note of a chord or its alteration has the potential of becoming a leading tone to a new key or its dominant.«(252)

54 Fétis 1844, 184-189.

55 Vgl. Rigaudière 2019, 171 f.

56 Gevaert 1907.

57 Ebd., 153, 213. 
Nach diesem inhaltlichen Kommentar der sehr reichen und gehaltvollen Publikation, empfiehlt sich, einige abschließende Bemerkungen zur Edition hinzuzufügen. Abgesehen von einigen Tippfehlern im Text, in den Anmerkungen und in der Bibliographie, ist es bedauerlich, dass die französischen Originaltexte der Zitate nicht zur Verfügung gestellt werden, handelt es sich doch um ein Buch, das sich in den französischsprachigen Ländern in Kürze sehr verbreiten wird.

Ebenso werden die Äquivalente der ins Englischen übersetzten Fachbegriffe nicht immer in der Originalsprache angegeben, was zu Unklarheiten führen kann, wie in der folgenden Passage: »[...] Kiesewetter noted that a number of troubadour and trouvère melodies seem to have been conceived within our smodern key system (Kiesewetter never seemed comfortable using the term tonalität in his own writing) [...].« (78) In diesem speziellen Fall wäre es nützlich gewesen den Ausdruck zu kennen, den Kiesewetter alternativ zu 'Tonalitätı verwendet hat.

\section{ZUSAMMENFASSUNG}

Mehr noch als eine Geschichte der >Tonalitäten zur Zeit Fétis' bietet dieses Buch ein reiches Panorama der musikwissenschaftlichen und -theoretischen Debatten, die durch den
Aufschwung der Musikgeschichtsschreibung im 19. Jahrhundert - wobei diese ihre Ambitionen in Zeit und Raum beträchtlich erweiterte auf ihrem Weg zu einer sallgemeinen Geschichtes - und durch die Restauration der Gregorianik, des Volksgesangs und der Orient-Studien ausgelöst wurden.

Die nicht unbedingt durch die Thematik vorgegebene Entscheidung, Fétis in den Mittelpunkt der Erzählung zu stellen, verleiht diesem überwältigenden historischen Fresko seinen Schwerpunkt. Es unterstreicht seine wissenschaftliche Allgegenwart und seine führende Rolle als Musikschriftsteller und Theoretiker, ohne zu verhehlen, dass bestimmte Autoren in einigen Bereichen von größerer Relevanz waren als er. ${ }^{58}$ Die Treue, mit der Fétis an einer Vorstellung von Tonalität festhält, die er einmal formuliert hat, und die bereits in den 1830er Jahren nahezu unverrückbar war, erklärt zweifellos die Hartnäckigkeit, mit der er manchmal fragile Thesen verteidigte, obwohl er doch von den Beiträgen seiner Kollegen hätte profitieren und seine Ideen regelmäßig hätte aktualisieren können.

übersetzt aus dem Französischen von Ariane Jeßulat

Marc Rigaudière

58 Christensen führt den sehr bezeichnenden Vergleich mit Kiesewetter an (175), welcher sich durch die Zusammenarbeit mit einem Spezialisten für die arabische Sprache und Geschichte abgesichert hatte, nämlich mit dem österreichischen Orientalisten Joseph von Hammer-Purgstall. Ein anderes Beispiel sind die skeptischen Einwände des Priesters Nicolaas Adrianus Janssen gegen Fétis' Idee, einige Regeln der musica ficta in den plaint-chant einzuführen. Fétis wird kein ernsthaftes Argument gegen diese Einwände finden (48 f.). 


\section{Literatur}

Barbereau, Auguste [1845], Traité d'harmonie théorique et pratique, 2 Bde., Paris: Schonenberger (auch Paris: Lemoine [o. J.]).

Barthes, Roland (1968), „L'effet de réel«, Communications 11, 84-89.

Battmann, Jacques-Louis (1855), Cours d'harmonie théorique et pratique appliqué spécialement à l'étude de l'accompagnement du plain-chant, Paris: Fleury.

Beaulieu, Désiré (1858), Mémoire sur quelques airs nationaux qui sont dans la tonalité grégorienne, Niort: Favre.

Berlioz, Hector (1862), À travers chants. Études musicales, adorations, boutades et critiques, Paris: Michel Lévy.

Bourgault-Ducoudray, Louis-Albert [1876], Trente mélodies populaires de Grèce et d'Orient, recueillies et harmonisées par L.-A. BourgaultDucoudray, Paris: Lemoine.

Christensen, Thomas (1985), Science and Music Theory in the Enlightenment. D'Alembert's critique of Rameau, Ph.D., Yale University.

Christensen, Thomas (1993), Rameau and Musical Thought in the Enlightenment, Cambridge: Cambridge University Press.

Christensen, Thomas (Hg.) (2002), The Cambridge History of Western Music Theory, Cambridge: Cambridge University Press.

Christensen, Thomas (2014), The Work of Music Theory: Selected Essays, London: Routledge.

Campos, Rémy (2013), François-Joseph Fétis musicographe, Genf: Droz.

Choron, Alexandre (1811), Considération sur la nécessité de rétablir le chant de l'Église de Rome dans toutes les Églises de l'Empire, Paris: Courcier.

Coussemaker, Charles Edmond Henri de (1856), Chants populaires des Flamands de France, Gand: Gyselynck.

Danjou, Félix (1845), »Revue de la musique religieuse, populaire et classique», Revue de la musique religieuse, populaire et classique 1, 5-16.

Derode, Victor (1828), Introduction à l'étude de l'harmonie, ou exposition d'une nouvelle théorie de cette science, Paris: Treutzel et Wurtz.

d'Ortigue, Joseph (1854), »Tonalité«, in: Dictionnaire liturgique, historique et théorique de plain-chant et de musique d'église au moyen âge et dans les temps modernes, Paris: Migne, 1446-1507.

Fétis, François-Joseph (1835), »Résumé philosophique de I'histoire de la musique», in: Biographie universelle des musiciens, Bd. 1, Brüssel: Leroux, xxxvii-ccliv.

Fétis, François-Joseph (1835-44 und 1860-67), Bibliographie universelle des musiciens et bibliographie générale de la musique, 1. Auflage, 8 Bde., Paris: Fournier und 2. Auflage, 8 Bde., Paris: Firmin Didot.

Fétis, François-Joseph (1840), Esquisse de I'histoire de l'harmonie, considérée comme art et comme science systématique, Paris: Bourgogne et Martinet.

Fétis, François-Joseph (1844), Traité complet de la théorie et de la pratique de l'harmonie, Paris: Schlesinger.

Fétis, François-Joseph (1859), Mémoire sur I'harmonie simultanée des sons chez les Grecs et les Romains, en réponse à la question suivante: Les Grecs \& les Romains ontils connu l'harmonie simultanée des sons?, Brüssel: Hayez.

Fétis, François-Joseph (1869-76), Histoire générale de la musique depuis les temps les plus anciens jusqu'à nos jours, 5 Bde., Paris: Firmin Didot.

Fétis, François-Joseph (2006), Correspondance, hg. von Robert Wangermée, Sprimont: Mardaga.

Gerbert, Martin (1784), Scriptores ecclesiastici de musica sacra potissimum. Ex variis Italiae, Galliae [et] Germaniae codicibus manuscriptis collecti et nunc primum publica luce donati, Bd. 2, Sankt Blasien: Typis SanBlasianis.

Gevaert, François-Auguste (1856), Méthode pour l'enseignement du plain-chant et la manière de l'accompagner, Paris: Girod. 
Gevaert, François-Auguste (1907), Traité d'harmonie théorique et pratique, $\mathrm{Bd}$. 2, Paris: Lemoine.

Gobineau, Joseph Arthur de (1853-55), Essai sur l'inégalité des races humaines, 4 Bde., Paris: Firmin Didot.

Groth, Renate (1983), Die französische Kompositionslehre des 19. Jahrhunderts, Wiesbaden: Steiner.

Herry, Jean (1842), Eunn dibab toniou evit kanaouennou santel ha gwersiou Breiz-Isel, Saint-Brieuc: Prud'homme.

Kastner, Georges (1857), Les voix de Paris. Essai d'une histoire littéraire et musicale des cris populaires de la capitale depuis le MoyenÂge jusqu'à nos jours, Paris: Brandus.

Kiesewetter, Raphael (1838), Ueber die Musik der neueren Griechen nebst freien Gedanken über altegyptische und altgriechische Musik, Leipzig: Breitkopf \& Härtel.

La Fage, Adrien de (1855-56), Cours complet de plain-chant, ou Nouveau traité méthodique et raisonné du chant liturgique de l'Église latine, à l'usage de tous les diocèses, Paris: Gaume frères.

Lesure, François (1974-76), „L'affaire Fétis«, Revue belge de musicologie 28/30, 214-221.

Milliot, Vincent (2016), »Les Cris de Paris, figures d'un peuple apprivoisé«, Revue de la Bibliothèque nationale de France 52, 12-25.
Münch, Marc-Mathieu (2004), L'effet de vie ou le singulier de l'art littéraire, Paris: Champion.

Niedermeyer, Louis / d'Ortigue, Joseph (1855), Traité théorique et pratique de l'accompagnement du plain-chant, Paris: Repos.

Nisard, Théodore (1860), Les vrais principes de l'accompagnement du plain-chant sur l'orgue, d'après les maîtres des XVe et XVIe siècles, Paris: Repos.

Rigaudière, Marc (2019), Les théories de I'harmonie et de la tonalité en France au $19^{e}$ siècle, Habil., Sorbonne Université.

Sanguinetti, Giorgio (2012), The Art of Partimento. History, Theory and Practice, New York: Oxford University Press.

Sorge, Georg Andreas (1747), Vorgemach der musicalischen Composition, Bd. 3, Lobenstein: Selbstverlag.

Tiersot, Julien (1889), Histoire de la chanson populaire en France, Paris: Plon.

Vincent, Alexandre (1854), De la musique des anciens Grecs. Discours prononcé au Congrès scientifique de France, Arras: Brissy.

Weber, Gottfried (1817), Versuch einer geordneten Theorie der Tonsetzkunst, Bd. 1, Mainz: Schott.

Weckerlin, Jean-Baptiste (1886), La chanson populaire, Paris: Firmin Didot.

Rigaudière, Marc (2020): Thomas Christensen, Stories of Tonality in the Age of François-Joseph Fétis, Chicago: University of Chicago Press 2019. ZGMTH 17/1, 155-165.

https://doi.org/10.31751/1039

(C) 2020 Marc Rigaudière (marc.rigaudiere@gmail.com)

Université Paris-Sorbonne; Institut de recherche en musicologie (IReMus)

Dieser Text erscheint im Open Access und ist lizenziert unter einer

Creative Commons Namensnennung 4.0 International Lizenz.

This is an open access article licensed under a

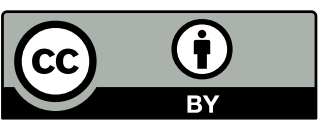

Creative Commons Attribution 4.0 International License.

eingereicht / submitted: 22/04/2020

angenommen / accepted: 22/04/2020

veröffentlicht / first published: 15/06/2020

zuletzt geändert / last updated: 15/06/2020 